\title{
Number of endothelial progenitor cells and microparticles are associated with body composition, but not fat intake or blood lipids
}

\author{
H. A. Altowaijri, M. Weech, K. Vafeiadou, E. Hobby, S.-Y. Wu, J. Madden, K. G. Jackson, \\ S. Todd, P. Yaqoob and J. A. Lovegrove \\ Department of Food and Nutrition Sciences, University of Reading, Whiteknights, PO Box 226, Reading RG6 6AP, UK
}

The endothelium plays an essential role in vascular homoeostasis and regulation of vascular tone. Endothelial dysfunction occurs in the early stages of atherosclerosis, and contributes to the formation of atherosclerotic plaques. Circulating markers of endothelial function include endothelial progenitor cells (EPC), which play a role in repair and maintenance of damaged endothelium ${ }^{(1)}$, and endothelial microparticles (EMP) and platelet microparticles (PMP) that are produced directly as a result of endothelial activation ${ }^{(2)}$. There is little data on the effects of dietary fat or body composition on these novel markers of endothelial function. The aim of this study was to examine the influence of habitual dietary fat intake, body composition and blood lipids on circulating EPC, EMP and PMP numbers in a cohort of human subjects with moderate cardiovascular risk.

Intakes of total fat, SFA, MUFA, $n-3$ PUFA and $n-6$ PUFA in the habitual diet of fifty-five volunteers were correlated with absolute numbers of blood EPC, EMP and PMP which were analysed using flow cytometry. EPC were stained with a combination of anti-CD34 and anti-CD309 (VEGFR2), and EMP and PMP were stained with anti-CD31 and anti-CD42b.

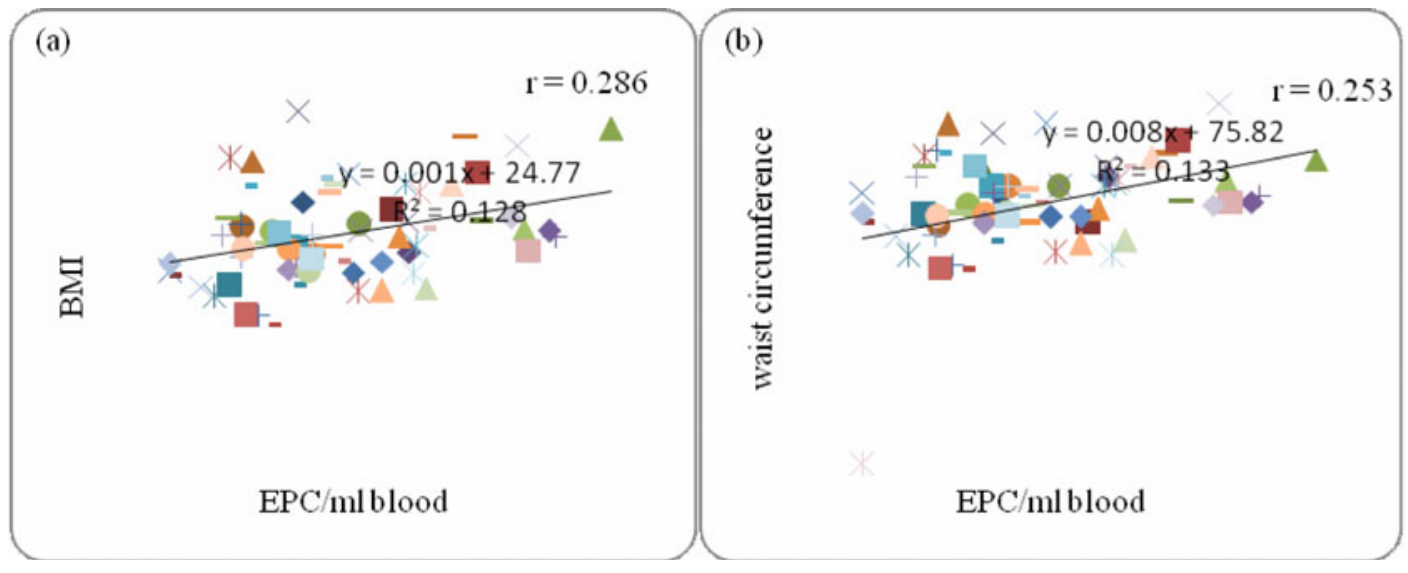

Fig. 1. Relationship between EPC number and (a) BMI and (b) waist circumference.

EPC numbers were positively correlated with BMI and waist circumference $(P=0.019,0.039$, respectively) (Figs. 1a and b). There were no other significant associations, and no relationship between dietary intake of fatty acids and EPC, EMP or PMP. There were no associations between EPC, EMP, PMP and blood lipids (cholesterol, HDL and TAG). Data from this study is not consistent with previous literature; most studies report an inverse association between adiposity and EPC numbers ${ }^{(3)}$. EPC numbers are also negatively associated with CVD risk ${ }^{(4)}$. Thus, this observation requires further investigation.

This work was funded by the Foods Standards Agency (grant number N02R0007002).

1. Rafil S \& Lyden D (2003) Nat Med 9, 702-712.

2. Diamant M, Tushuizen ME, Sturk A et al. (2004) Eur J Clin Invest 34, 392-401.

3. Hill JM, Zalos G, Halcox JP et al. (2003) $N$ Engl J Med 348, 593-600.

4. Müller-Ehmsen J, Braun D, Schneider T et al. (2008) Eur Heart J 29, 1560-1568. 